\title{
Standard Transformations for the Normalization of ER Schemata
}

\author{
Otto Rauh ${ }^{1}$ and Eberhard Stickel ${ }^{2}$ \\ ${ }^{1}$ Fachhochschule Heilbronn, Daimlerstr. 35, D-74653 Künzelsau \\ ${ }^{2}$ Europa-Universität Viadrina, Große Scharrnstr. 59, D-15230 Frankfurt (Oder)
}

\begin{abstract}
Normalization, which makes up the core of the design theory for relational databases, is also considered an important technique to improve the quality of ER schemata. We first present a framework for describing ER schema transformations. Then a normal form, ER-BCNF ${ }^{\text {null }}$, is defined which corresponds to BCNF but takes null values into account. Finally, a set of transformations is suggested which might be used to achieve this normal form.
\end{abstract}

\section{Introduction}

Normalization has been accepted as an important property of Entity-Relationship (ER) schemata improving the clarity of these schemata and avoiding both data inconsistencies and costly data manipulations in the databases derived from them (cf. e.g. Batini 1992). There is an elaborate normalization theory for relational databases that may also be utilized for ER modelling. Unfortunately, relational normalization algorithms do not go very well with ER modeling, at least if we want to preserve the stepwise and intuitive design process which is typical for the ER approach. In contrast, a method based on standard transformations is far better suited. Standard transformations rely on a classification of unfavourable constellations. For certain patterns of such situations, transformations are described in an abstract manner. The designer who is faced with an unfavourable situation identifies this situation with one of these patterns and then takes the action suggested.

There has been valuable preliminary work on the subject, going into two directions. Kobayashi (1986), Rosenthal (1988) and Hainaut (1991, 1993) contributed to the elaboration of the basic principles. Chung (1981), Ling (1985a, 1985b) and Batini (1992) suggested standard transformations for different purposes. Nevertheless, until now the subject has not gained the attention it deserves and needs more working out.

We shall first present a general framework for describing ER schema transformations. Then normal forms for ER schemata will be defined and a set of standard transformations will be presented to achieve them. Finally, we shall give an outlook on further work on the subject which is necessary. 


\section{A Framework for Describing ER Schema Transformations}

\subsection{Schema Description}

Exact description of ER schemata is a prerequisite for describing schema transformations. Our schema descriptions will consist of two parts: (i) a description of schema structure, written in a simple language, (ii) a set of constraints. We shall make use of ER diagrams as well, but only for the purpose of illustration. The following example describes the structure of a schema $S_{1}$ :

$S_{1}$ : EntitySet Employee (Attributes EmpID, EmpName not null; Identifier EmpID);

EntitySet Department (Attributes DeptID, DeptName; Identifier DeptID); RelationshipSet MemberOf (Participants (Employee, Employee, $(1,1)$ ), (Department, Department, $(0, \mathrm{n}))$ );

There is an entry for each construct, i.e. each entity set and each relationship set, of the schema. A relationship set entry contains a three-tuple for every participating entity set (participant), consisting of its name, its role within the relationship set, and its cardinality. If the entity set in question participates only once in the relationship set the role name may be the same as the entity name. Sometimes there are attributes too in a relationship set entry, but not in this example. Domain declarations for attributes, which would be necessary in a practical schema declaration, are omitted to keep the description simple.

There are basically two types of constraints that influence the values of a database. Implicit constraints are consequences of structure and its semantics. Attributes, for instance, must always be functionally dependent on the identifier, and identifier attributes have only definite values. Explicit constraints are not implied by structure and thus have to be declared separately. We use a descriptive language, called ERC (Entity-Relationship Calculus) to express arbitrary constraints for ER schemata (cf. Rauh 1994). ERC is mainly based on relational tuple calculus (TRC) which has already been proposed by Codd (1972).

Constraints in ERC are always closed formulas in the sense that every variable is bound by a quantifier. Thus a truth value can be assigned to such a rule with respect to a certain database instance. Let us assume one such rule, stating that department names have to be unique:

$$
\begin{gathered}
\left(\forall d_{1}, d_{2}\right)\left(\text { Department }\left(d_{1}\right) \wedge \text { Department }\left(d_{2}\right) \wedge \neg\left(d_{1}==d_{2}\right)\right. \\
\left.\rightarrow d_{1}[\text { DeptName }] \neq d_{2}[\text { DeptName }]\right)
\end{gathered}
$$

The double equality sign "==" is used to denote identity of entity sets or relationship sets. The familiar equality sign $"={ }^{6}$ is applied only to express equality of attribute values.

For some kinds of constraints, e.g. functional dependencies (FD), where there are special notations, these notations will be used if they are more convenient. In particular for the schemata used in this paper, these special notations will suffice. 


\subsection{Basic Concepts of Schema Transformations}

Let $S$ be an ER schema. Then with $s(S)$ we denote a database instance that is structured according to $S$ and meets its constraints. A schema transformation $T$ is a pair $\left(\left(S_{1}, S_{2}\right), t\right)$, where $S_{1}$ is the source schema, $S_{2}$ denotes the target schema, and $t$ is a list of assignments converting a database instance $s_{1}\left(S_{1}\right)$ into a database instance $s_{2}\left(S_{2}\right)$. According to Hainaut (1993), the first element of $T$, i.e. $\left(S_{1}, S_{2}\right)$, is called the structural mapping, whereas $t$ is the instance mapping. Formulating an instance mapping for a certain structural mapping $\left(S_{1}, S_{2}\right)$ assigns a meaning to the components of the target schema $S_{2}$ which are fed with data from $s_{1}\left(S_{1}\right)$ by $t$. Thus we may call $t$ the semantics of the transformation (Hainaut 1993). There might be many instance mappings for a certain structural mapping.

Not necessarily, an instance mapping has to provide an assignment for every component of the target schema, neither is it necessary that the whole source database is transferred to the target database. We are, however, especially interested in transformations which convert data without loss of information. In order to be able to describe such transformations, we introduce the concept of reversible transformation.

A schema transformation $T=\left(\left(S_{1}, S_{2}\right), t\right)$ is reversible if there exists a transformation $W=\left(\left(S_{2}, S_{1}\right), w\right)$ such that an arbitrary database instance $s_{1}\left(S_{1}\right)$ may be converted into an instance $s_{2}\left(S_{2}\right)$ by using $T$, and $s_{2}$ may be re-converted into $s_{1}$ by using $W$ afterwards. $W$ is called an inverse transformation to $T$ (cf. Hainaut 1993).

Apart from those special cases when a schema containing too much information is reduced, we should demand reversibility for any transformation a schema is undertaken to improve its quality.

\subsection{Instance Mappings}

An instance mapping consists of a set of assignments which have the general form

$$
<\text { schema component }>:=<\text { query }>\text {, }
$$

where < schema component $>$ is an entity or relationship set of the target schema, and $<$ query $>$ is a database query referring to the components of the source schema. In this paper, we shall use ERC to formulate such queries. There are two basic forms of assignments to entity sets:

$$
\begin{aligned}
& <\text { entity set name }>:=\quad\left\{\left\{<\text { entity variable }>\left.\right|^{\prime}<\text { condition }>{ }^{\prime}\right\}\right. \\
& <\text { entity set name }>:=\quad\left\{\left(\left\{<\text { value list }>\left.\right|^{\prime}<\text { condition }>\right\}^{\prime}\right.\right.
\end{aligned}
$$

The first one is really a short form. It may be used if an entity set is not changed during the transformation, except perhaps for its name. We have to use the second form if this condition does not hold, maybe because the entity set in the target schema has another set of attributes than that in the source schema or because it is composed of several constructs of the source schema. In this case the attribute values which are the elements of < value list > must match the list of attributes in the declaration of the entity set. For each value of the identifier of this entity set, a new entity is supposed to be constructed on the basis of the assignment. 
There are also two basic forms for relationship set assignments, depending on whether the relationship set in question has attributes or not:

$$
\begin{aligned}
& <\text { relationship set name }>:=\quad\left\{\text { l }^{2}<\text { participant list }>\left.\quad\right|^{\prime}<\text { condition }>\text { ' }\right\} \\
& <\text { relationship set name }>:=\quad\left\{<\text { participant list }>\text {; }<\text { value list }>\text { I }^{\prime}\right. \\
& <\text { condition }>\text { ' }\} \text { '. }
\end{aligned}
$$

Therein $<$ participant list $>$ is a list of entity variables which take a special form if the entity set in question is not taken over unchanged from the source schema. Then $<$ entity variable > consists of an entity designator, which is a function assigning an entity to a list of attribute values that are interpreted as its identifier values. An entity designator has the general form < entity set name $>$ ( $<$ attribute variable list $>$ ), where $<$ attribute variable list $>$ takes the values of the identifier's attributes. If the relationship set in question has any attributes the second of the two forms has to be taken, and the attributes have to be listed in < value list >.

We continue our example and define a second schema $S_{2}$ :

$S_{2}$ : $\quad$ EntitySet Employee (Attributes EmpID, EmpName, EmpDept not null; Identifier EmpID).

For a transformation $U=\left(\left(S_{2}, S_{1}\right), u\right)$, a suitable instance mapping $u$ is

$$
\text { Employee }:=\{e[E m p I D], e[\text { EmpName }] \mid \text { Employee }(e)\}
$$

Department $:=\{e[$ EmpDept $]$, null $\mid$ Employee $(e)\}$

MemberOf $:=\{$ Employee $(e[$ EmpID $])$, Department $(e[$ EmpDept $]) \mid$ Employee $(e)\}$.

In line three, entity designators are used to denote the entities of types Employee and Department which participate in MemberOf relationships. A transformation in the other direction is $V=\left(\left(S_{1}, S_{2}\right), v\right)$, where $v$ is

$$
\begin{aligned}
\text { Employee }:= & \{e[\text { EmpID }], e[\text { EmpName }], d[\text { DeptID }] \mid \text { Employee }(e) \wedge \\
& \text { Department }(d) \wedge(\exists m)(\text { MemberOf }(m) \wedge \\
& m: \text { Employee }==e \wedge m \text { Department }=d)\} .
\end{aligned}
$$

In this assignment, a participant function like $m$ :Department represents the participant in a relationship which plays the role denoted behind the colon.

For such small examples reversibility is easy to assess. $U=\left(\left(S_{2}, S_{1}\right), u\right)$ is a reversible transformation as $V=\left(\left(S_{1}, S_{2}\right), v\right)$ is a transformation inverse to $U$. In contrast to $U, V$ is not reversible. Attribute DeptName of entity set Department in $S_{1}$ is not contained in the result part of the query in the only assignment $v$ consists of. Moreover, there is also no other reversible transformation since there is no attribute in $S_{2}$ that could take the values of DeptName. 


\section{Standard Transformations for Normalization}

\subsection{Normal Forms for ER constructs}

In relational database design, third normal form (3NF) is generally considered a sufficient condition for a table if functional dependencies (FD) are the only dependencies to be taken into account. Among the different forms of 3NF, Boyce-Codd normal form (BCNF) is probably the most popular and plausible. Surely, not every possible BCNF database has the property of preserving all functional dependencies (cf. Ullman 1988, p. 404). But as this is only a theoretical possibility and hardly a practical one, there is no reason why we should not choose BCNF as the desired structure for ER constructs. We use a definition given by Gardarin $(1989$, p. 164) to characterize BCNF: A relation scheme $R$ is said to be in Boyce-Codd normal form $(B C N F)$ if, for all disjoint nonempty sets $X$ and $Y$ in $R$, if $X \rightarrow Y$ then $X$ is a superkey for $R$.

Unfortunately, null values are ignored in this definition and other definitions of BCNF. Let $R(A, B, C)$ be a relation scheme and $F=\{A \rightarrow B, B \rightarrow C\}$ a set of FD's in $R$. Then normalization algorithms demand decomposition into $(A, B)$ and $(B, C)$, tacitly assuming that if $B$ is null then $C$ is null as well. If this was not the case $B$ could not serve as a primary key in the second scheme. Rather than using decomposition $\{(A, B),(A, C)\}$ instead, which has other shortcomings, we shall use a weaker form of $B C N F$, called $B C N N^{\text {null }}$, that tolerates such situations:

A relation scheme $R$ is said to be in $\mathrm{BCNF}^{\text {null }}$ if whenever $X \rightarrow Y, X \cap Y=\varnothing$, holds in $R$, and $Y$ is always null if $X$ is null, then $X$ is a superkey for $R$.

It is easy to see that a relation scheme in $B C N F$ is in $\mathrm{BCNF}^{\text {null }}$ as well.

To be able to apply BCNF and $\mathrm{BCNF}^{\text {null }}$ (and arbitrary normal forms) to ER constructs, we use the concept of construct relation scheme (CRS). Let $K$ be a construct of an ER scheme and all attributes of $K$ be atomic. The construct relation scheme for $K$, written $C R S(K)$, is formed according to the following rules:

- If $K$ is an entity set then $C R S(K)$ contains all the attributes of $K$. The identifier of $K$ becomes the primary key of $C R S(K)$.

- If $K$ is a relationship set then $C R S(K)$ has the following set of attributes: the attributes of $K$, if there are any, plus the union of the identifiers of all the participants of $K$. All these identifiers are declared as foreign keys, and all foreign keys are declared "not null". The primary key of $C R S(K)$ consists of the union of the identifiers of the identifying participants.

Normal forms for ER databases are based on construct relation schemes: Let $K$ be an ER construct. Then $K$ is in ER-BCNF (ER-BCNF ${ }^{\text {null }}$ ) if $C R S(K)$ is in BCNF $\left(B C N F^{\text {null }}\right)$. Similar definitions may be formulated for all normal forms of relational database theory.

We shall use both ER-BCNF and ER-BCNF ${ }^{\text {null }}$ to judge whether a certain construct requires normalization or not. Which of both forms applies depends on the situation. 
In general, we may say that, in a completely normalized schema, every construct meets at least ER-BCNF ${ }^{\text {null }}$.

\subsection{The Basic Conception for ER Normalization}

Our approach to ER modelling is strongly influenced by the analysis approach to BCNF normalization (cf. Ullman 1988, p. 403). Just like in relational normalization, unfavourable constructs are decomposed, the major difference being that the connections between the parts are not maintained by foreign keys but in a manner which is adequate for ER. If the violation of ER-BCNF or ER-BCNF ${ }^{\text {null }}$ was caused by only one functional dependency one transformation suffices, otherwise transformations have to be applied repeatedly until no harmful dependency is left. As every transformation removes one of the harmful dependencies and does not add new ones the process terminates. In the end, no harmful dependency is left.

Transformations to achieve ER-BCNF ${ }^{\text {null }}$ or ER-BCNF have a common property which normalization transformations striving for higher normal forms do not possess. Suppose $K$ is a construct violating ER-BCNF or ER-BCNF ${ }^{\text {null }}$. Then one of the constructs created during the transformation, we call it normally $K^{\prime}$, has the same identifier as $K$. More precisely, $C R S(K)$ has the same primary key as $C R S(K)$. We shall call such transformations detachments, in contrast to splitting transformations, where this property does not hold.

Despite some common features, there are also significant differences between our approach and relational normalization:

- When decomposing an entity set we always preserve the identifier of the original construct $K$ when it is changed to $K^{\prime}$, at the most it might be renamed. This is because, unlike a key of a relation, an identifier of an entity set is explicitly defined by the designer; it is not just one of the candidate keys.

- In contrast to relational normalization, ER normalization alone cannot guarantee a nonredundant and reasonably structured schema. Whereas relational normalization takes the whole of the schema as input, the application of normal forms within ER modeling is only local (cf. Rauh 1993). We can, for instance, convert every entity set into constructs meeting ER-BCNF or ER-BCNF ${ }^{\text {null }}$, but there might still be double entity sets in the schema, perhaps under different names. This is why an ER schema should have the porperty of minimality in addition to the property of normality (see Batini 1992, Rauh 1993 for details).

\subsection{Transformation of Entity Sets (Transformation N1)}

For the sake of simplicity, we take the same name for a construct and for the set of its attributes during the following treatment of standard transformations. Similarly, the name of a CRS is also used for its set of attributes. The meaning should always be clear from the context. We shall use stars $\left({ }^{*}\right)$ for lower or upper bounds of cardinalities if their values are of no importance in the special context. Throughout the discussion, we will assume that all attributes of a CRS are functionally dependent on the primary key. Thus only additional functional dependencies will be mentioned explicitly. Furthermore, we shall follow a constant pattern in our discussion of trans- 
formations. First, a description of the (unfavourable) initial situation is given, and the transformation is portrayed in an informal manner. We thereby address structural and instance mappings at the same time by discussing how the attributes of the source schema are to be shifted to the target schema. Then a formal description of the source and target schemata is provided to define the structural mapping, and the instance mapping is given as a set of ERC assignments.

Before we describe how an unfavourable entity set may be transformed into more favourable constructs, let us first state in which situations normalization is necessary: In general, an entity set requires normalization if it violates ER-BCNF ${ }^{\text {null }}$.

Initial situation: Let $K$ be an entity set with the set of attributes $K$ and the identifier $S, S \subset K . K$ is not in ER-BCNF ${ }^{\text {null }}$ as there is a FD $X \rightarrow Y$, with $X \subset K, Y \subset K$ and $X \cap$ $Y=\varnothing$, where $X$ is not a superkey for $C R S(K)$, and $Y$ is null if $X$ is null. $K$ is linked to an entity set $L$ with identifier $C$ by a relationship set $K L$. We have to consider two variants

a) $\quad$ is functionally dependent on $X$, and if $X$ is null then $C$ is null as well.

b) The condition assumed for a) does not hold.

There is an important implication following from this description: in variant a) the upper bound of $K L$ 's cardinality on the side of $K$ must be 1 as $C$ is functionally dependent on $X$, and $X$, being a subset of $K$, is dependent on the identifier $S$.

Please notice that there is no loss of generality in assuming that, in the initial situation, $K$ is connected with only one other entity set. If there are other relationship sets they are treated accordingly.
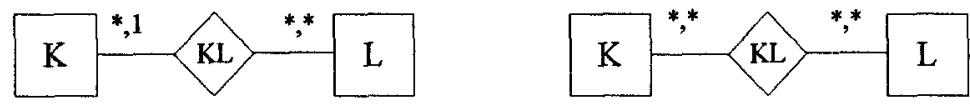

Fig. 1: Source schemata for N1, variant a) left, variant b) right

Informal Description of Transformation: An new entity set $N$ with the set of attributes $N=\{X, Y\}$ and the identifier $X$ is created. Entity set $K$ is changed to $K^{\prime}$ by removing those attributes of $X$ and $Y$ that are not contained in the identifier of $K$. Consequently, $K^{\prime}$ has the set of attributes $K^{\prime}=(K \backslash(Y \backslash S)) \backslash(X \backslash S)=(K \backslash(X \cup Y)) \cup$ $S$, with $S$ being the identifier. $K^{\top}$ and $N$ are connected by a new relationship set $K N$, the cardinalities of which are determined by the following rules: If $X$ has been declared not null within $K$, the cardinality on the side of $K^{\prime}$ is $(1,1)$, otherwise it is $(0,1)$. The cardinality on the side of $N$ is set to $(0, n)$ provided that there are no further constraints suggesting another choice. How $L$ is to be connected with the other entity sets of the target schema depends on the variant. If variant $b$ ) applies the former connection with $K$ is retained under the new name $K L$, with $K^{\prime}$ as participant instead of $K$. In case of variant a) $K L$ is dropped, and a new relationship set $N L$ 
between $N$ and $L$ is created. Its cardinality on the side of $N$ is the same as the cardinality on the side of $K$ in the former relationship set $K L$, i.e. $(0,1)$ or $(1,1)$. For the side of $L,(0, n)$ must be taken unless additional constraints suggest another choice.
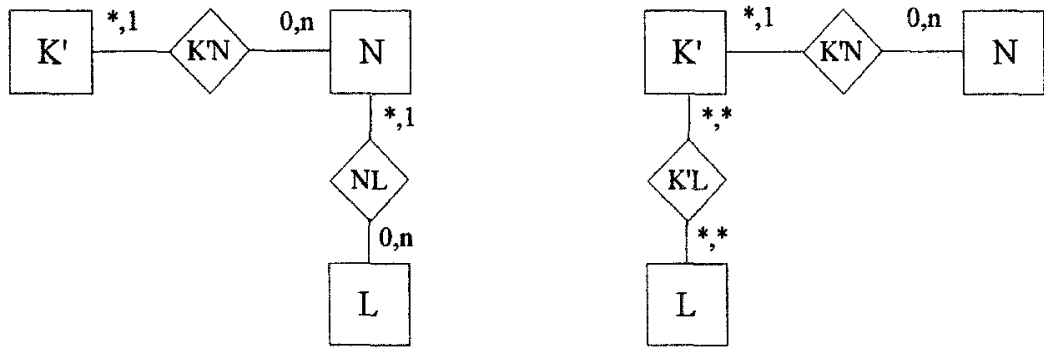

Fig. 2: Target schemata for N1, variant a) left, variant b) right

Structural Mapping: The source schemata are nearly identical for variants a) and b). We begin with a). To avoid confusion, we shall write $A \rightarrow B$, if $B$ is functionally dependent on $A$, and $A \Rightarrow B$ for $\neg A \vee B$ :

EntitySet $K(K ;$ Identifier $S) ; S, X, Y \subset K ; X \rightarrow Y, \neg(X \rightarrow K) ; X=$ null $\Rightarrow Y=$ null

EntitySet $L$ ( $L$; Identifier $C$ ); $C \subseteq L, X \rightarrow C ; X=$ null $\Rightarrow C=$ null

RelationshipSet $K L\left((K, K,(*, 1)),\left(L, L,\left({ }^{*}, *\right)\right)\right)$.

For variant b), we get

EntitySet $K(K$; Identifier $S) ; S, X, Y \subset K ; X \rightarrow Y, \neg(X \rightarrow K) ; X=$ null $\Rightarrow Y=$ null

EntitySet $L(L$; Identifier $C) ; C \subseteq L$

RelationshipSet $K L\left(\left(K, K,\left({ }^{*},{ }^{*}\right)\right),\left(L, L,\left({ }^{*}, *\right)\right)\right)$.

The target schema for a) is

EntitySet $K^{\prime}\left(K^{\prime} ;\right.$ Identifier $\left.S\right) ; S \subseteq K^{\prime}$

EntitySet $N(X, Y$; Identifier $X)$

EntitySet $L(L$; Identifier $C) ; C \subseteq L$

RelationshipSet $K^{\top} N\left(\left(K, K^{\top},\left({ }^{*}, 1\right)\right),(N, N,(0, n))\right)$

RelationshipSet $N L((N, N,(*, 1)),(L, L,(0, n)))$.

For b), we drop the declaration for $N L$ and add the following declaration instead:

RelationshipSet $K^{\prime} L\left(\left(K^{\prime}, K^{\prime},\left({ }^{*},{ }^{*}\right)\right),\left(L, L,\left({ }^{*},{ }^{*}\right)\right)\right)$. 
Instance Mapping: For variant a), we get:

$$
\begin{aligned}
K^{\prime} & :=\{k[(K \backslash(X \cup Y)) \cup S] \mid K(k)\} \\
N & :=\{k[X], k[Y] \mid K(k) \wedge k . X \neq n u l l\} \\
L & :=\{l \mid L(l)\} \\
K^{\prime} N & :=\left\{K^{\prime}(k[S]), N(k[X]) \mid K(k) \wedge k \cdot X \neq n u l l\right\} \\
N L & :=\{N(k[X]), l \mid K(k) \wedge L(l) \wedge(\exists k l)(K L(k l) \wedge k l: K==k \wedge k l: L==l)\}
\end{aligned}
$$

Instead of $N L, K^{\prime} L$ is needed for variant b):

$K^{\prime} L:=\left\{K^{\prime}(k[S]), l \mid K(k) \wedge L(l) \wedge(\exists k l)(K L(k l) \wedge k l: K==k \wedge k l: L==l)\right\}$.

\subsection{Transformation of Relationship Sets (Transformation N2)}

A relationship set $K$ requires normalization if one of the following conditions holds

1. $K$ violates $\mathrm{ER}-\mathrm{BCNF}^{\text {null }}$,

2. $K$ violates ER-BCNF because of a harmful dependency $X \rightarrow Y$ in $C R S(K)$, and $X$ is functionally dependent on a proper subset of the set of participant identifiers of $K$.

Initial Situation: A relationship set $K$ with the set of attributes $K, K \neq \varnothing$, and the participants $P_{1}, P_{2}, \ldots, P_{\mathrm{n}}$ which may be all or in part identifying for $K . K$ is not in ER-BCNF as there is a FD $X \rightarrow Y, Y \subset K$ and $X \cap Y=\varnothing$, in $C R S(K)$ where $X$ is not a superkey for $C R S(K)$. For simplicity, we write $R K$ for both $C R S(K)$ and its attributes. Suppose $S_{1}, S_{2}, \ldots, S_{\mathrm{n}}$ are the identifiers of the participants. Then the set of attributes is $R K=K \cup S_{1} \cup S_{2} \cup \ldots \cup S_{\mathrm{n}} . X$ and $Y$ must be proper subsets of $R K$. There are three variants which have to be considered. The first two of them correspond with condition 2 mentioned above:

a) $X$ is, either trivially or nontrivially, functionally dependent on exactly one of the participant identifiers $S_{1}, S_{2}, \ldots, S_{\mathrm{n}}$ in $R K$.

b) $K$ has an arity greater than two and there is a full FD of $X$ on the union of at least two of the identifiers $S_{1}, S_{2}, \ldots, S_{\mathrm{n}}$.

c) Neither a) nor b) apply, but if $X$ is null then $Y$ is null.

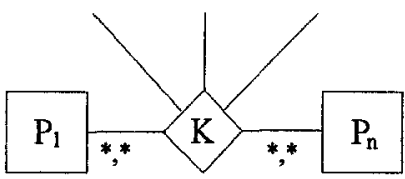

Fig. 3: Source schema for N2 
Remark: For variants a) and b), already a violation of ER-BCNF is seen as a reason for transforming $K$ as there are entity sets that can absorb $\{X, Y\}$, so that $X$ is not needed as an identifier. This is not the case for $c$ ), where only a violation of weaker ER-BCNF ${ }^{\text {null }}$ causes a transformation, and an infringement of ER-BCNF must be tolerated. The additional condition $X=n u l l \Rightarrow Y=n u l l$ is set to mark the violation of weaker ER-BCNF ${ }^{\text {null }}$.

Informal Description of transformation, variant a): Let us assume that $X$ is dependent on the identifier $S_{m}$ of participant $P_{m}$. We change the name of $K$ into $K^{\prime}$ and remove $Y$ and the subset of $X$ that has been contained in it. Thus $K$ has attributes $K^{\prime}=K \backslash(X \cup Y) . P_{m}$ is changed to $P_{m}^{\prime}$ and enriched by the additional attributes $X$ and $Y$, as far as they are not already there. Consequently, $P_{m}{ }^{\prime}$ has the attributes $P_{m}$, $A=X \backslash P_{m}$, and $B=Y \backslash P_{m}$.

Structural Mapping, variant a): In the source schema declaration, there is an entry for every entity set $P_{i}, i=1,2, \ldots, n$. Similarly, the declaration for $K$ contains every $P_{i}$ as a participant.

EntitySet $P_{i}\left(P_{i}\right.$, Identifier $\left.S_{i}\right) ; S_{i} \subseteq P_{i}$

RelationshipSet $K\left(\left(P_{i}, P_{i},\left({ }^{*},{ }^{*}\right)\right) ; K\right)$;

In addition, the following constraints are valid in the source schema: $S_{m} \rightarrow X ; X \rightarrow$ $Y ; Y \subset K ; X \subset\left(K \cup P_{m}\right)$. In the target schema, $P_{m}$ becomes $P_{m}^{\prime}$ and gets a separate declaration. For all other $P_{i}, i=1,2, \ldots, m-1, m+1, \ldots, n$, the declaration remains the same:

EntitySet $P_{i}\left(P_{i} ;\right.$ Identifier $\left.S_{i}\right) ; \quad S_{i} \subseteq P_{i}$

EntitySet $P_{m}^{\prime}\left(P_{m}, A, B\right.$; Identifier $\left.S_{m}\right) ; \quad S_{m} \subseteq P_{m}^{\prime}$

RelationshipSet $K^{\prime}\left(\left(P_{1}, P_{1},\left({ }^{*},{ }^{*}\right)\right), \ldots,\left(P_{m-1}, P_{m-1},\left({ }^{*},{ }^{*}\right)\right),\left(P_{m}{ }^{\prime}, P_{m}{ }^{\prime},\left(^{*},{ }^{*}\right)\right)\right.$, $\left.\left(P_{m+1}, P_{m+1},\left({ }^{*}, *\right)\right), \ldots,\left(P_{n}, P_{n},\left({ }^{*},{ }^{*}\right)\right) ; K^{\prime}\right)$
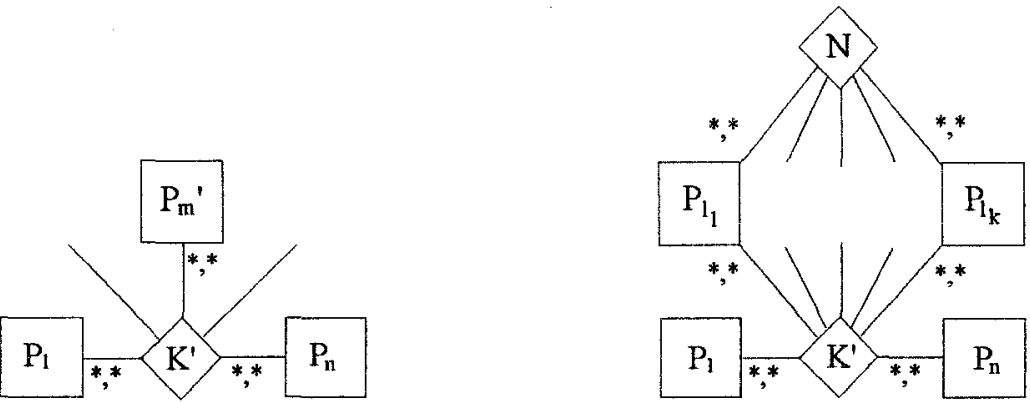

Fig. 4: Target Schemata for N2, variant a) left, variant b) right 
Instance Mapping for variant a): As in the description of the target schema, $P_{m}{ }^{\prime}$ is treated separately:

$$
\begin{aligned}
P_{i}:= & \left\{p \mid P_{i}(p)\right\}, \quad i=1,2, \ldots, m-1, m+1, \ldots, n \\
P_{m}^{\prime}:= & \left.\left\{p \mid P_{m}\right], k\left[X \backslash P_{m}\right], k\left[Y \backslash P_{m}\right] \mid P_{m}(p) \wedge K(k) \wedge k: P_{m}==p\right\} \\
K^{\prime}:= & \left\{p_{1}, p_{2}, \ldots, p_{n} ; k[K \backslash(X \cup Y)] \mid P_{1}\left(p_{1}\right) \wedge P_{2}\left(p_{2}\right) \wedge \ldots \wedge P_{n}\left(p_{n}\right)\right. \\
& \left.\wedge k: P_{1}=p_{1} \wedge k: P_{2}=p_{2} \wedge . . \wedge k: P_{n}==p_{n}\right\}
\end{aligned}
$$

Notice that, if $X$ is not contained in $K$, then $k[X]$ refers to an empty set of attributes, and so does $k\left[X \backslash P_{m}\right]$.

Informal Description of Transformation, variant $\mathbf{b}$ ): Suppose that $X$ is fully functionally dependent on $S_{l_{1}} \cup S_{l_{2}} \cup \ldots \cup S_{l_{k}}$ where $S_{l_{i}}$ is the identifier of participant $P_{l_{i}}, i=1,2, \ldots, k$. Then a new relationship set $N$ is created between $P_{l_{1}}, P_{l_{2}}, \ldots, P_{l_{k}} . N$ gets those subsets of $X$ and $Y$ as attributes that have been contained in $K$, and consequently has attributes $N=(X \cap K) \cup Y$. The cardinalities of $N$ are the same as those of $K$ in the same position unless there are additional constraints suggesting another choice. $X$ and $Y$ are removed from $K$ as far as they have been there. $K$ becomes $K^{\prime}$, now having the set of attributes $K^{\prime}=K \backslash(X \cup Y)$.

Structural Mapping, variant b): Apart from explicit constraints, the source schema is the same as for $a)$ :

EntitySet $P_{i}\left(P_{i}\right.$, Identifier $\left.S_{i}\right) ; \quad S_{i} \subseteq P_{i}$

RelationshipSet $K\left(\left(P_{i}, P_{i},\left({ }^{*},{ }^{*}\right)\right) ; K\right)$;.

Constraints to consider are: $\left\{P_{l_{1}}, P_{l_{2}}, \ldots, P_{l_{k}}\right\} \rightarrow X ; X \rightarrow Y ; Y \subset K ; X \subset(K \cup$ $S_{l_{1}} \cup S_{l_{2}} \cup \ldots \cup S_{l_{k}}$ ). Compared to variant a), the target schema is enlarged by the declaration for relationship set $N$ :

EntitySet $P_{i}\left(P_{i}\right.$; Identifier $\left.S_{i}\right) ; \quad i=1,2, \ldots, n ; S_{i} \subseteq P_{i}$

RelationshipSet $K^{\prime}\left(\left(P_{i}, P_{i},\left({ }^{*},{ }^{*}\right)\right) ; K^{\prime}\right) ; \quad i=1,2, \ldots, n$;

RelationshipSet $N\left(\left(P_{l_{i}}, P_{l_{i}},\left({ }^{*}, *\right)\right) ; N\right) ; \quad i=1,2, \ldots, k$

\section{Instance Mapping, variant b):}

$$
\begin{aligned}
P_{i}:= & \left\{p \mid P_{i}(p)\right\}, \quad i=1,2, \ldots, n \\
K^{\prime}:= & \left\{p_{1}, p_{2}, \ldots, p_{n} ; k[K \backslash(X \cup Y)] \mid P_{1}\left(p_{1}\right) \wedge P_{2}\left(p_{2}\right) \wedge \ldots \wedge P_{n}\left(p_{n}\right)\right. \\
& \left.\wedge k: P_{1}==p_{1} \wedge k: P_{2}==p_{2} \wedge \ldots \wedge k: P_{n}==p_{n}\right\} \\
N:= & \left\{p_{1}, p_{2}, \ldots, p_{k} ; k[(X \cap K) \cup Y] \mid P_{l_{1}}\left(p_{1}\right) \wedge P_{l_{2}}\left(p_{2}\right) \wedge \ldots \wedge P_{l_{k}}\left(p_{k}\right)\right. \\
& \left.\wedge K(k) \wedge k: P_{l_{1}}=p_{1} \wedge k: P_{l_{2}}==p_{2} \wedge \ldots \wedge k: P_{l_{k}}=p_{k}\right\}
\end{aligned}
$$

Informal Description of Transformation, variant c): Let $P_{j_{1}}, P_{j_{2}}, \ldots, P_{j_{u}}$ be the identifying partipicants of $K$ and $S_{j_{1}}, S_{j_{2}}, \ldots, S_{j_{u}}$ be their identifiers. $K$ is changed 
into an entity set $K^{\prime}$ with attributes $\left\{D_{1}=S_{j_{1}}, D_{2}=S_{j_{2}}, \ldots, D_{u}=S_{j_{u}}, E=K \backslash X, F=\right.$ $K \backslash Y\}$ and identifier $\left\{D_{1}, D_{2}, \ldots, D_{u}\right\} . K$ is connected with the former participants of $K$ by $n$ binary relationship sets. Their cardinalities on the side of $K^{\prime}$ are all $(1,1)$. On the side of the former participants the cardinalities of $K$ in the same positions are taken. A new entity set $N$ is created with attributes $\{X, Y\}, X$ being the identifier. In the source schema, $X$ may have been overlapping with one or more identifiers of the participants. Let us assume that $P_{x_{1}}, P_{x_{2}}, \ldots, P_{x_{r}}$ are those participants, and $A_{1} \subseteq S_{x_{1}}$, $A_{2} \subseteq S_{x_{2}}, \ldots, A_{r} \subseteq S_{x_{r}}$ are the attributes overlapping with $X$. Then $X=A_{1} \cup A_{2} \cup \ldots \cup$ $A_{r} \cup\left(X \backslash\left(S_{x_{1}} \cup S_{x_{2}} \cup \ldots \cup S_{x_{r}}\right)\right)$. As we want $N$ to consist of the attributes $\{X, Y\}$, we set $N=\left\{A_{1}, A_{2}, \ldots, A_{r}, B=X \backslash\left(S_{x_{1}} \cup S_{x_{2}} \cup \ldots \cup S_{x_{r}}\right), Y\right\} . N$ is connected with $K^{\prime}$ by a binary relationship set $K^{\prime} N$ which has no attributes. With respect to the cardinalities of $K N$ the following rules are applied: The upper bound on the side of $K$ is 1 since there is at most one $X$-value for a certain $K^{\prime}$-entity. For the lower bounds, we take 0 if nulls have been allowed for $X$ within $K, 1$ if not. On the side of $N$, the cardinality is $(1, n)$ since $X$ and $Y$ were originally defined within $K$ and could not exist without an instance of $K$ and, on the other hand, certain values of $X$ may well occur together with different instances of $K$.

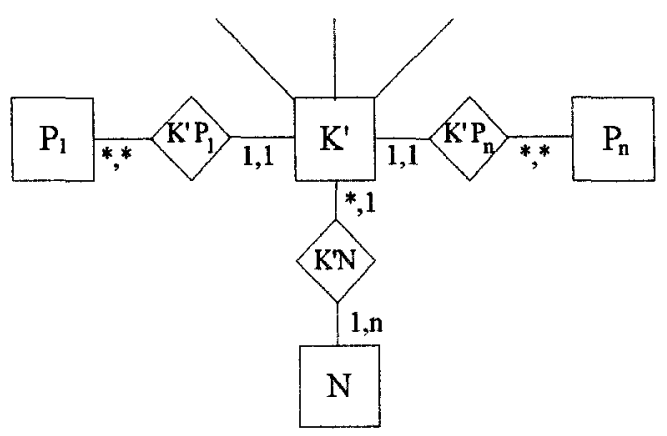

Fig. 5: Target schema for N2, variant c)

Structural Mapping for variant c): The source schema is the same as for a) and $b$ ), except for the constraints, which are: $X \rightarrow Y ; Y \subset K, X \subset\left(K \cup P_{1} \cup \ldots \cup P_{n}\right)$. Now the target schema contains two new entity sets $K^{\prime}$ and $N, n$ binary relationship sets $K^{\prime} P_{i}$ and a new relationship set $K^{\prime} N$ :

EntitySet $P_{i}\left(P_{i}\right.$; Identifier $\left.S_{i}\right) ; \quad i=1,2, \ldots, n ; S_{i} \subseteq P_{i}$

EntitySet $N\left(A_{1}, A_{2}, \ldots, A_{r}, B, Y\right.$; Identifier $\left.A_{1}, A_{2}, \ldots, A_{r}, B\right)$

EntitySet $K^{\prime}\left(D_{1}, D_{2}, \ldots, D_{u}, E, F\right.$; Identifier $\left.D_{1}, D_{2}, \ldots, D_{u}\right)$

RelationshipSet $K^{\prime} P_{i}\left(\left(K^{\prime}, K,(1,1)\right),\left(P_{i}, P_{i},\left({ }^{*},{ }^{*}\right)\right) ; \quad i=1,2, \ldots, n\right.$

RelationshipSet $K^{\prime} N\left(\left(K^{\prime}, K^{\prime},(1,1)\right),(N, N,(1, n))\right.$. 
Instance Mapping for variant $c)$ :

$$
\begin{aligned}
P_{i}:= & \left\{p \mid P_{i}(p)\right\}, \quad i=1,2, \ldots, n \\
N:= & \left\{p_{1}\left[S_{x_{1}} \cap X\right], p_{2}\left[S_{x_{2}} \cap X\right], \ldots, p_{r}\left[S_{x_{r}} \cap X\right], k\left[X \backslash \left(\left(S_{x_{1}} \cap X\right) \cup\left(S_{x_{r}} \cap X\right) \cup\right.\right.\right. \\
& \left.\left.\ldots \cup\left(S_{x_{r}} \cap X\right)\right)\right], k[Y] \mid K(k) \wedge P_{x_{1}}\left(p_{1}\right) \wedge P_{x_{2}}\left(p_{2}\right) \wedge \ldots \wedge P_{x_{r}}\left(p_{r}\right) \wedge k: P_{x_{1}}=p_{1} \wedge \\
& \left.k: P_{x_{2}}==p_{2} \wedge \ldots \wedge k: P_{x_{r}}==p_{r}\right\} \\
K^{\prime}:= & \left\{p_{1}\left[S_{j_{1}}\right], p_{2}\left[S_{j_{2}}\right], \ldots, p_{u}\left[S_{j_{u}}\right], k[K \backslash X], k[K \backslash Y)\right] \mid P_{j_{1}}\left(p_{1}\right) \wedge P_{j_{2}}\left(p_{2}\right) \wedge \ldots \wedge \\
& \left.P_{j_{u}}\left(p_{u}\right) \wedge X(k) \wedge k: P_{j_{1}}=p_{1} \wedge k: P_{j_{2}}==p_{2} \wedge \ldots \wedge k: P_{j_{u}}==p_{u}\right\} \\
K^{\prime} P_{i}:=\{ & K^{\prime}\left(p_{1}\left[S_{j_{1}}\right], p_{2}\left[S_{j_{2}}\right], \ldots, p_{u}\left[S_{j_{u}}\right]\right), p \mid P_{j_{1}}\left(p_{1}\right) \wedge P_{j_{2}}\left(p_{2}\right) \wedge \ldots \wedge P_{j_{u}}\left(p_{u}\right) \wedge \\
& \left.P_{i}(p) \wedge(\exists k)\left(K(k) \wedge k: P_{j_{1}}==p_{1} \wedge k: P_{j_{2}}==p_{2} \wedge \ldots \wedge k: P_{j_{u}}==p_{u}\right)\right\} \\
K^{\prime} N:= & \left\{K^{\prime}\left(p_{1}\left[S_{j_{1}}\right], p_{2}\left[S_{j_{2}}\right], \ldots, p_{u}\left[S_{j_{u}}\right]\right), N\left(p_{1}\left[S_{x_{1}} \cap X\right], p_{2}\left[S_{x_{2}} \cap X\right], \ldots, p_{r}\left[S_{x_{r}} \cap X\right],\right.\right. \\
& \left.\left.k\left[X \backslash\left(S_{x_{1}} \cup S_{x_{2}} \cup \ldots \cup S_{x_{r}}\right)\right]\right) \mid P_{x_{1}}\left(p_{1}\right) \wedge P_{x_{2}}\left(p_{2}\right) \wedge \ldots \wedge P_{x_{r}}\left(p_{r}\right) \wedge K(k)\right\}
\end{aligned}
$$

\section{Summary and Outlook}

A general framework for the description of ER schema transformations has been introduced and then been applied to describe a set of standard transformation to achieve an ER normal form derived from BCNF, but taking null values into account.

The results presented mark only the first step within a greater project to develop standard transformations to improve the quality of ER designs. Much work has still to be done. For instance, normalization up to fifth normal form, which is not covered by this paper, will be dealt with in a future publication. In addition, there are many interesting but unsolved questions concerning the properties of schema transformations. A significant problem is, for example, how reversibility can be assessed for arbitrary transformations. An approach presented by Makowsky (1986) in another context may be used for this purpose: The basic principle is to translate an ER schema into a relational database scheme, called ER-compatible relational database scheme (ERS), that reflects its structure so closely that both schemata might be used as substitutes for each other. The ERS of an ER schema consists of the CRS of all its constructs. Hence there is a one to one correspondence between relation schemes and ER constructs. We can then show how the ERS of the target schema is produced from the ERS of the source schema using a series of lossless database operations. The concept of lossless decomposition, which is closely related to the concept of reversibility, can be used as an aid. Unfortunately, relational database theory provides only a limited basis for judging upon losslessness if null values are not neglected (cf. Maier 1983, Ullman 1988, Atzeni 1993). Thus some basic work has to be done previously. 


\section{References}

Atzeni, P., De Antonellis, V. (1993). Relational Database Theory, Redwood City, California: Benjamin/Cummings

Batini, C., Ceri, S., Navathe, S. (1992). Conceptual Database Design: An EntityRelationship Approach, Redwood City, California: Benjamin/Cummings

Chung, I., Nakamura, F., Chen, P. (1983). A Decomposition of Relations Using the Entity-Relationship Approach. Proc. of the 2nd International Conference on EntityRelationship Approach, Washington, D.C. 1981, North-Holland, 1983

Codd, E, (1972). Relational Completeness of Data Base Sublanguages. In: R. Rustin (ed.), Data Base Systems, Englewood Cliffs, New Jersey: Prentice-Hall

Gardarin, G. and Valduriez, P. (1989). Relational Databases and Knowledge Bases, Reading, Mass.: Addison-Wesley

Hainaut, J.-L. (1990). Entity-Relationship Models: Formal Specification and Comparison. In H. Kangassalo (ed.), Proceedings of the 9th International Conference on the Entity-Relationship Approach, Lausanne, Switzerland, 1990

Hainaut, J.-L., Tonneau, C., Joris, M., Chandelon, M. (1993). Schema Transformation Techniques for Database Reverse Engineering. In: Proceedings of the 12th International Conference on Entity-Relationship Approach, Arlington, Texas, Dec 15-17, 1993

Jajodia, S., Ng, P.A. (1983). The Problem of Equivalence for Entity-Relationship Diagrams. IEEE Transactions on Software Engineering 9 (1983), No. 5, pp. 617-630

Kobayashi, I. (1986). Losslessness and Semantic Correctness of Database Schema Transformations: Another Look of Schema Equivalence. Information Systems, Vol. 11, No. 1, pp.41-59, 1986

Ling, T.W. (1985a). A Normal Form for Entity-Relationship Diagrams. Proc. of the 4th International Conference on Entity-Relationship Approach, Chicago 1985, North-Holland

Ling, T.W. (1985b). An Analysis of Multivalued and Join Dependencies Based on the Entity- Relationship Approach. Data \& Knowledge Engineering 1 (1985), pp. 253-271

Maier, D. (1983). The Theory of Relational Databases, Rockville, Md: Computer Science Press

Makowsky, J., Markowitz, V., Rotics, N. (1986). Entity Relationship Consistency for Relational Schemas. In G. Ausiello and P.Atzeni (eds.), ICDT '86, Springer-Verlag

Rauh, O. and Stickel, E. (1993), Searching for Compositions in ER Schemes. In R. Elmasri, V. Kouramajian (eds.), Proceedings of the 12th Int' Conference on EntityRelationship Approach, Arlington, TX, 1993

Rosenthal, A., Reiner, D. (1988). Theoretically Sound Transformations for Practical Database Design. Proc. of the 6th International Conference on Entity-Relationship Approach, New York 1987, pp. 115-132, North-Holland

Ullman, J. (1988). Principles of Database and Knowledge-Base Systems, Volume I, Rockville, Maryland: Computer Science Press 